\title{
Macrophage activation syndrome: A diagnostic challenge (Review)
}

\author{
ANCA BOJAN $^{1,2}$, ANDRADA PARVU ${ }^{1,2}$, IULIA-ANDREA ZSOLDOS ${ }^{2}$, \\ TUNDE TOROK ${ }^{1,2}$ and ANCA DANIELA FARCAS ${ }^{3,4}$ \\ ${ }^{1}$ Hematology Department, 'Iuliu Hatieganu' University of Medicine and Pharmacy; \\ ${ }^{2}$ Hematology Department, 'Prof. Dr. Ioan Chiricuta' Oncological Insitute, 400124 Cluj-Napoca; \\ ${ }^{3}$ Internal Medicine Department, 'Iuliu Hatieganu' University of Medicine and Pharmacy; \\ ${ }^{4}$ Cardiology Department, Emergency County Clinic Hospital, 400006 Cluj-Napoca, Romania
}

Received March 26, 2021; Accepted April 26, 2021

DOI: $10.3892 / \mathrm{etm} .2021 .10336$

\begin{abstract}
Macrophage activation syndrome (MAS) represents an acute and severe inflammatory syndrome, idiopathic (primary) or secondary to infections, rheumatic diseases, malignancies, or drugs. MAS is underdiagnosed, being confused with sepsis, adverse effects of anti-arthritic drugs or exacerbated symptoms of evolving rheumatologic or infectious diseases. Because of the late diagnosis, most patients do not benefit from effective therapy, leading to death. Elucidation of valid early diagnostic criteria of MAS would be a particularly important step in reducing the mortality due to this pathology. Thus, the purpose of this review based on 40 studies centered on the diagnostic criteria of MAS. We detailed the main diagnostic criteria and the few diagnostic scores or sets of criteria that have been recently published. The criteria most frequently encountered in the literature include: Fever, hepatosplenomegaly, hyperferritinemia, hepatopathy, coagulopathy, thrombocytopenia, hypertriglyceridemia, decrease in erythrocyte sedimentation rate and bone marrow hemophagocytosis. The most elaborate diagnostic score will result following an ongoing international project and consensus, the Delphi International Survey.
\end{abstract}

\section{Contents}

1. Introduction

2. Justification and objectives of the literature review

3. Review methods

4. Data analysis and results

5. Discussion

6. Conclusions

Correspondence to: Dr Andrada Parvu, Hematology Department, 'Iuliu Hatieganu' University of Medicine and Pharmacy, Nr. 73, 21 Decembrie 1989 Boulevard, 400124 Cluj-Napoca, Romania E-mail: parvuandrada@hotmail.com

Key words: macrophage activation syndrome, hemophagocytosis, pancytopenia, inflammatory syndrome, diagnosis score

\section{Introduction}

Macrophage activation syndrome (MAS) is a severe inflammatory systemic abnormality with lethal potential characterized by pancytopenia, coagulopathy, hepatopathy, neurological disorders and hemophagocytosis. The inflammation is caused by the uncontrolled activation of macrophages and T cells (1-3). The symptomology of this syndrome is serious and potentially lethal. Mortality has been reported in $20-53 \%$ of cases (4-6). The incidence is estimated to be about $1.2 / 1$ million individuals in Sweden and one in 100,000 in Texas, USA. Moreover, the prevalence of fulminant MAS in patients with systemic juvenile idiopathic arthritis (sAJIs) is $10 \%(1,2,7)$.

Monocyte-macrophage series physiology. Macrophages are immune mediators, particularly important and very sensitive to humoral stimuli. Macrophages are large cells (60-80 microns) and have high enzyme content. Their major role is to maintain inflammation [by secretion of interleukin (IL)-1, IL-8, IL-12, tumor necrosis factor (TNF)- $\alpha$, plasmin, transferrin], antibacterial and antitumor action with the production of oxidative lesions. Macrophages can phagocyte foreign particles or apoptotic cells (1-3).

Mosser and Edwards (3) proposed a classification of the macrophage population based on three main homeostatic activities: Immune defense, inflammation, and immune regulation. Macrophages are extremely heterogeneous and under the action of humoral factors, they can express markers from another category at the membrane level while taking on other functions.

Interstitially fixed macrophages are called histiocytes (liver Kupffer cells, pulmonary and alveolar macrophages, microglia cells in the central nervous system, spleen, serous, connective tissue and hematogenous marrow). Histiocytes represent the first line of defense at the time of inflammation (1-3).

Mobile macrophages in the bloodstream are called monocytes. They can migrate into tissues, where their transformation into histiocytes plays a role in phagocytosis. Monocyte recruitment into tissues is mediated by lymphokines: Interferon (INF) $-\gamma$ and TNF- $\alpha$. Natural killer (NK) cells secrete INF- $\gamma$ but do not produce a constant amount capable of sustaining an activated macrophage population. In contrast, $\mathrm{T}$ helper 1 (LiTH1) lymphocytes are capable of continuous INF- $\gamma$ secretion and maintenance of macrophage activation. Macrophage 
interaction with LiTH1 is essential because it lays the basis for cell-mediated immunity. The proinflammatory cytokines secreted by post-activation macrophages play an important role in defending the host but can also lead to serious injuries if the inflammatory process is not adequately controlled $(8,9)$.

MAS pathophysiology. Primary MAS is triggered by the excessive proliferation of LiTH1 which is caused by the decrease/lack of NK cell cytotoxicity, a decrease due to a mutation in the gene that encodes perforin (a protein that plays a role in the cytotoxicity of NK cells and $\mathrm{CD}^{+}$cytotoxic T lymphocytes). Perforin is involved in the apoptosis of tumor or viral infected cells and controls cell proliferation. Due to the decrease in perforin levels and the lack of NK cell activity, lymphocytes are persistently activated and secrete two major macrophage activators: INF- $\gamma$ and granulocyte-macrophage colony-stimulating factor (GM-CSF). Stimulated by these two mediators, macrophages activate and proliferate uncontrollably (8-17).

MAS-causing/triggering factors. MAS appears most often as a complication of inflammatory systemic diseases, collagenosis (especially juvenile inflammatory arthritis, but also systemic lupus erythematosus, Kawasaki disease, sarcoidosis, dermatomyositis, Still's disease, Sjogren's disease) (18-29), infection (fungal, parasitic, viral, bacterial, zoonotic (30-34) or secondary of cancers (35-37).

There have been reports of cases of MAS following treatment with various drugs such as non-steroidal anti-inflammatory drugs (NSAIDs), gold salts, sulfasalazine or methotrexate, adalimumab (recombinant human TNF- $\alpha$ monoclonal antibody), tocilizumab (human IL-6), and etoposide (38-42). The infectious and drug-inducing factors of MAS are presented in Table I (34).

MAS terminology and framing. The term 'macrophage activation syndrome' was first used by Stephan et al in 1993 (18). MAS was characterized by Hadchouel et al in 1985 in the form of a hemorrhagic syndrome associated with neurological and hepatic changes, a syndrome observed in 7 patients with idiopathic juvenile arthritis (43). MAS can occur at any age and does not have gender or race specificity.

There is an inconsistency in the literature regarding MAS framing, mostly because MAS is a borderline syndrome between hematology, immunology, rheumatology, and infectious diseases, so that specialists from all of these domains have published data concerning MAS (44-49).

Some authors consider it to be a self-standing syndrome, trying to define the correct diagnosis and treatment algorithm; others advocate for its inclusion in the category of histiocytosis. Histiocytosis diseases are characterized by proliferation and accumulation of macrophages and dendritic cells, which may be primary and secondary, benign and malignant (Table II; classification of histocytic disorders) $(7,32,43,50,51)$. In this classification, MAS is equivalent to hemophagocytic syndromes that may be primary or secondary. We consider this definition of MAS as the most complex and correct, being supported by several groups of authors $(32,44,45)$.

Other authors consider MAS to be an acquired entity of hemophagocytic limphohistiocytosis (LHL), secondary only to inflammatory and autoimmune diseases (Table III) (44,52-54).
The development of a universal MAS diagnosis and treatment method will be difficult as there is no unanimously accepted terminology. This also occurs because of the heterogeneity of this syndrome (primary, secondary to infection, inflammation or neoplasia) $(34,36,55)$. Multiple authors consider MAS to be quite similar to hemophagocytic lymphohistiocytosis (HLH), both clinically and according to laboratory investigations $(5,8,44,48,56)$. Other authors use HLH only for familial HLH (primary), with secondary HLH called MAS (39), or do not use the term MAS, considering it as synonymous with all HLHs $(19,20,35,43)$. Thus, again, development of a universal MAS diagnosis and treatment method will be difficult as long as there is no unanimously accepted terminology.

Clinical and laboratory manifestations of MAS. The ilness generally starts acutely,clinically/biologically with a sepsis-like syndrome. Patients have high fever, secondary pancytopenia, symptoms due to hepatosplenomegaly and hepatic impairment, lymphadenopathy and hyperferritinemia. Coagulation is often abnormal consisting in the prolongation of prothrombin time (PT) and of activated partially thromboplastin time (aPTT) and decreased fibrinogen. Purpura or hemorrhages can also be present. Neurological symptoms consist of headache, temporospatial disorientation, irritability, convulsions or coma. The anatomopathological analysis of the bone marrow is important for diagnosis. This analysis shows numerous macrophages with phagocytic hematopoietic cells, which explains pancitopenia. All manifestations lead to multiple organ failure and ultimately to patient death $(1,2,7,19,33,44,57)$.

\section{Justification and objectives of the literature review}

The goal of MAS treatment is to suppress hyperinflammation and remove the cause of the disease (removal of stimuli that maintain ineffective activation of macrophages and T lymphocytes). Although multiple treatment methods of MAS have been proposed according to its etiology (immunosuppressants, monoclonal antibodies, etiological treatment), late diagnosis prevents effective therapy, leading to death $(7,50)$. Many authors believe that MAS is underdiagnosed, being confused with sepsis, adverse effects of anti-arthritic drugs or exacerbated symptoms of evolving rheumatologic diseases. Not being diagnosed on time and treated appropriately, the condition of the patients deteriorates (58).

Elaboration of early valid diagnostic criteria of MAS would be a vital step in reducing mortality due to this pathology. Moreover, it would be useful to define a short list of triage screening to identify patients predisposed to develop MAS. Due to all of this and to the fact that genetic tests of NK cell cytotoxicity are not available on a routine basis, it is vital that the diagnostic or the screening paraclinical examinations are simple and accessible for any hospital (58).

The objective of this study was to carry out a systematic review of the current literature on the correct diagnosis of MAS.

\section{Review methods}

Defining criteria for inclusion and exclusion of studies. The inclusion criteria for the studies in this systematic analysis were Romanian or English language studies, macrophage activation 
Table I. Triggers of macrophage activation syndrome (34).

Trigger

Viral infections

Bacterial infections

Fungal infections

Parasitic infestations

Drugs
Specific agents

Cytomegalovirus, herpes simplex virus, Epstein-Barr virus, Varicella-zoster virus, adenovirus, influenza virus, Dengue virus, Parvovirus B19, Coxsackie-virus

Enterobacteriaceae, Salmonella, Haemophilus, Pneumococcus, Mycobacteria, Mycoplasma, Brucella, Staphylococcus

Candida, Histoplasma, Cryptococcus

Leishmania, Pneumocystis carinii

Sulphasalazine, aspirin, morniflumate, indomethacin, NSAIDs, penicillamine, methotrexate, gold salts, etanercept, phenytoin, intravenous soluble lipids

NSAIDs, nonsteroidal anti-inflammatory drugs.

Table II. Classification of histiocytic disorders $(7,36)$.

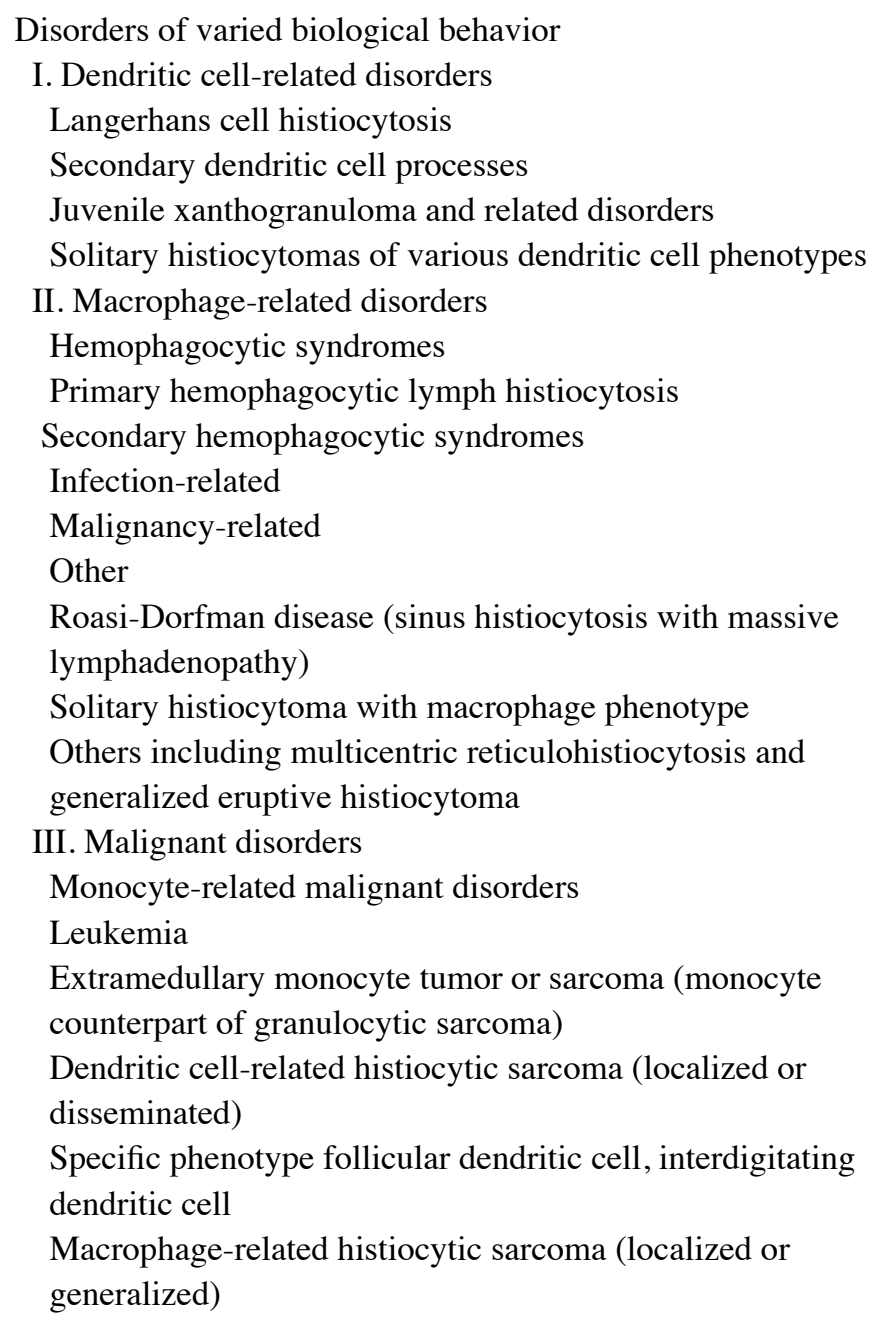

studies, studies focused on MAS diagnosis, recent studies published after 2001, ISI-rated publications and indexed in BDI databases.

Exclusion criteria included studies that could not be accessed in extenso, MAS studies, but non-focused on diagnostic criteria, studies that did not recognize MAS as a clinical entity, and fundamental research studies on animals.
Table III. Classification and underlying conditions of HLH (44).

\author{
Genetic HLH \\ Familial HLH (Farquhar disease ${ }^{\mathrm{a}}$ ) \\ Known gene defects (perforin, munc 13-4, syntaxin 11) \\ Unknown gene defects \\ Immune deficiency syndromes \\ Chédiak-Higashi syndrome (CHS) \\ Griscelli syndrome (GS) X-linked lymphoproliferative \\ syndrome (XLP) \\ Acquired HLH \\ Exogenous agents (infectious organisms, toxins) \\ Infection-associated hemophagocytic syndrome (IAHS) \\ Endogenous products (tissue damage, metabolic products) \\ Rheumatic diseases \\ Macrophage activation syndrome (MAS) \\ Malignant diseases
}

${ }^{a}$ Familial HLH was first described by Farquhar and Claireaux in 1952.

Searches in the MEDLINE database were conducted using the following methodology:

Advanced search: [('macrophage activation syndrome' [MeSH Terms] OR ('macrophage' [All Fields] AND 'activation' [All Fields] AND 'syndrome' [All Fields]) OR 'macrophage activation syndrome' [All Fields]) AND ('diagnosis' [Subheading] OR 'diagnosis' [All Fields] OR 'diagnosis' [MeSH Terms])) AND ('loattrfree full text'[sb] AND ('2001/01/01'[PDAT]: '2014/09/20'[PDAT])] with the filter enabled for fully accessible studies published since 2001/01/01 to date. A total of 114 studies resulted from this search.

We also researched for further reference among the bibliographic sources of the studied articles. Thus, 28 studies were considered relevant in the field; they met the inclusion criteria and were considered.

The search in the AIDSline database, following the methodology described above, returned 18 results.

The search in the Cancerlit database was performed using the following parameters: 'Macrophage activation syndrome 
diagnosis' or 'macrophage activation syndrome treatment'. It did not return any results.

Embase database searching returned 4 results, but the full text was not available for free.

The search in the Cochrane database was performed with the term 'macrophage activation syndrome' and we used as sub-headings the terms 'diagnosis' or 'therapy'. It did not return any results.

Selection of studies. The total number of studies identified was 164. Following the reading and analysis of the data from these studies, 124 studies were excluded, based on the inclusion or exclusion criteria previously listed, so that the systematic analysis included 40 studies.

The first MAS studies were published in the 20th century. Hadchouel et al (43) were among the first who discovered this syndrome without naming it. They characterized it as a complex pathology consisting of three syndromes: Hemorrhagic, neurologic and hepatocitolysis. Stephan et al (18) assigned it the name 'macrophage activation syndrome' and published 4 cases. These studies were not included in the analysis and were published before 2001 .

Gathering data from studies. For the data from the analyzed studies to be extracted uniformly and impartially, we created an electronic data collection form containing the following parameters: Eligibility criteria, design of the study, the population included in the study (diagnosis, number of subjects), the purpose of the published article. In this electronic form, we attached tables, figures and images that could also be included in the final text. For each study included in the systematic analysis, we completed such a form.

\section{Data analysis and results}

We analyzed the 40 studies selected according to the previously described criteria. The main sets of diagnostic criteria of MAS found in the articles included in the review are discussed, but before that, the clinical symptoms and laboratory analyses that led to the compilation of these diagnostic algorithms will be analyzed.

The MAS clinical and paraclinical criteria used in the 40 studies included in this systematic review are produced by the pro-inflammatory and anti-inflammatory cytokines secreted in MAS. The main diagnostic criteria found in the studied articles are as follows.

Hyperferritinemia. Hyperferritinemia in MAS can be explained by the expression of CD163 membrane protein of hemophagocytic macrophages. The role of CD163 is to bind the hemoglobin-haptoglobin complex and to protect cells from oxidative stress-induced by free hemoglobin resulting from the digestion of macrophage phagocytic erythrocytes. After hemoglobin phagocytosis, hemoglobin is decomposed inside the macrophage to bilirubin, carbon monoxide and free iron. Free iron is scized into the siderotic granules inside its macrophage or transported to the hematogenous marrow where it is distributed to the red cell precursors. The more the macrophages enclose more iron, the higher the amount of intracellular siderotic granules. The serum ferritin is increased directly in proportion to the amount of iron in the macrophages. The minimum level of ferritin required to diagnose lymphohistiocytosis or MAS is $500 \mu \mathrm{g} / \mathrm{l}$. The level of ferritin in MAS may be over 5,000 $\mu \mathrm{g} / 1$. Thus, a much higher ferritin (over 10,000 $\mathrm{ng} / \mathrm{dl}$ ) is an important diagnostic criterion in MAS. Ravelli (36) wanted to propose hyperferritinemia as the sole diagnostic criteria of MAS, but there were cases of idiopathic juvenile arthritis with high ferritin values. On the other hand, some authors observed that ferritinemia over 10,000 ng/dl also occurs in other diseases such as AIDS, neoplasms, fulminant hepatic diseases and idiopathic hemosiderosis $(5,7,34,36,59,60)$.

Emmenegger et al even proposed the introduction of high ferritin values as a screening method for MAS along with histopathological evidence of hemophagocytosis (61).

Fever. Fever is a feature of the inflammatory syndrome, and is produced by cytokines and TNF secreted by both NK cells and lymphocytes which activate macrophages. Although it can be highlighted in all MAS patients, it cannot be considered a symptom specific to this condition. However, in combination with other more or less specific criteria, fever can become a valid diagnostic criteria for MAS $(1,2,7,19,31,33,41,42,45)$.

Hepatopathy. Hepatic damage through increased transaminases may be present in many pathologies. Like fever, this criterion cannot be considered specific as long as it is solitary and is not in association with other relevant criteria $(1,2,7,19,31,43,62)$.

Hypertriglyceridemia. In addition, similar to hepatocytolysis, an increased level of triglyceride is not specific to MAS, but can be a useful criterion as part of a set of diagnostic criteria. Hypertriglyceridemia can be considered as a positive diagnostic criterion for MAS after a differential diagnosis with other clinical situations where it could be judged in the context of other laboratory tests $(46,54)$.

Hemophagocytosis. Histopathological evidence of hemophagocytosis cannot be a single diagnostic criterion for MAS, but can be helpful in confirming the syndrome when the diagnosis is difficult to establish. Hemophagocytosis, pathognomonic for MAS, may not be present at the beginning of symptomatology. It can also be highlighted in other sites such as the liver, spleen, and lymph nodes $(5,7,31,33,41)$.

Decrease in erythrocyte sedimentation rate. Decrease in the erythrocyte sedimentation rate is also characteristic of MAS. This may actually reflect hypofibrinogenemia secondary to fibrinogen consumption and hepatic dysfunction. A decrease in the rate of erythrocyte sedimentation could differentiate MAS from a worsening of inflammation in chronic rheumatoid disease. Suspicion of MAS may appear when the erythrocyte sedimentation rate is low and ferritin and D-dimers are elevated. Follistatin-like protein 1, ferritin and erythrocyte sedimentation rate may become MAS biomarkers. Their modification may be correlated with the expression of certain genes $(5,7,16,55)$.

Pancytopenia. MAS affects at least two cell lines, but most of the time all three are affected. In MAS secondary to infections 
or inflammatory diseases, the levels of leukocytes and platelets may be initially increased due to the underlying disease; a decrease in the two cell lines is evident and pronounced after a certain time of evolution $(19,31,33,42,43)$. Hemophagocytosis is not the only mechanism for the occurrence of pancytopenia in MAS; the 'storm' of secreted cytokines causes inhibition of hematopoiesis $(4,10)$.

Coagulopathy. Coagulopathy is characteristic of MAS and has been reported in many studies. This is expressed by the prolongation of the PT and aPTT. Fibrinogen is low. Activated macrophages determine through cytokines the increase in plasminogen activator level, which causes hyperfibrinolysis to amplify the hemodialysis syndrome produced by disseminated intravascular coagulation (DIC) $(5,7,10,21,31,33,43,47)$.

Decrease in NK cell cytotoxicity. The absence of NK cell activity and high IL-2 receptor-soluble $\alpha$-chain values are parameters proposed to be part of the set of criteria for MAS. These tests are unable to be performed in all laboratories as routine tests (57). The applicability of these criteria to patients with MAS associated with lupus is also problematic. In lupus, autoimmune cytopenia and immunosuppression are common and cannot be differentiated from MAS (23). In these patients, hyperferritinemia and lactate dehydrogenase may aid in the differential diagnosis $(59,60)$.

sCD25 and sCD163 as MAS markers. Among the clinical characteristics of MAS chosen by the international study community, there are markers such as soluble CD25 (sCD25) and soluble CD163 (sCD163). Researchers believe that they could be the key to differentiating MAS from other pathologies. CD25 is a soluble IL-2 receptor subunit. CD163 is a transmembrane protein present in haemophagocytic macrophages. CD163 binds the hemoglobin-haptoglobin complex and protects the cells against oxidative stress. The plasma levels of these two compounds reflect the degree of activation and expansion of $\mathrm{T}$ lymphocytes and macrophages $(3,11,15)$.

It should be noted that the first 9 criteria consist of one clinical criterion (fever) and 8 simple laboratory criteria that are available to any hospital service, which is why they are preferred to be included in a diagnostic score, unlike the last two exposed criteria: Decreased NK and sCD25 and sCD163 cytotoxicity, which cannot be routinely assessed.

The main sets of criteria/diagnostic scores proposed by different groups of authors for MAS diagnosis are further evaluated as follows.

First, for identifying a MAS diagnostic algorithm, Stabile et al (34) reviewed literature data on the frequency of different symptoms, signs or biological parameters that occurred in patients diagnosed with MAS. They used as a bibliographic source the studies of four reputable teams in the field of lymphohistocytosis research: Sawhney et al (6), Stephan et al (18), Emmenegger et al (51) and Ravelli (36). The most common symptoms that occur in MAS patients according to these authors include fever, splenomegaly, coagulopathy, thrombocytopenia, hepatopathy, hyperferritinemia and hemophagocytosis.

Secondly, Sawhney et al presented 9 cases of MAS which they encountered during 20 years of experience. Out of a total of 143 patients with rheumatologic diseases, a total of 9 out of 9 patients had fever, 8 out of 9 presented with hepatosplenomegalies, 7 out of 9 had bone marrow hemophagocytosis, 6 out of 9 presented with lymphadenopathies, 6 out of 9 had hepatopathies, and 5 out of 9 presented with coagulopathy; these were Sawney's proposed criteria for an MAS-positive diagnosis (6).

Third, the authors of another study followed 7 patients with MAS associated with juvenile chronic arthritis. The MAS diagnosis was based on the following clinical and laboratory criteria: Fever, thrombocytopenia, hepatosplenomegaly, pancytopenia, increased erythrocyte sedimentation rate, coagulopathy and hypofibrinogenemia. Bone marrow biopsy was performed for all the patients and hemophagocytosis was evidenced at 5 patients (15).

Fourth, the first MAS diagnostic set was developed in 2005 , but it had many limitations. The criteria were not subsequently validated in clinical activity. The set of criteria referred to i) clinical criteria: Central nervous system symptoms (irritability, disorientation, lethargy, headache, epileptic seizures, coma), hemorrhagic syndrome (purpura, ecchymosis, mucosal bleeding), hepatomegaly ( $>3 \mathrm{~cm}$ under the rib cage); ii) laboratory criteria: Leukopenia, thrombocytopenia, hepatocitolysis, hypofibrinogenemia; iii) histopathological criterion: Hemophagocytosis on bone marrow specimen (47).

Fifth, an extensive international collaborative expert project began in 2010 to reach a consensus on MAS diagnostic criteria (63). The project was carried out in four stages. Stage 1 was the Delphi International Survey, which was distributed to rheumatology, internal medicine, hematology, intensive care specialists (of the 505 physicians who received the survey, 232 completed the data and provided feedback). Stage 2 was an international collection of data on patients with MAS associated with rheumatologic disease or other medical conditions that can be confused with MAS. Stage 3 consisted of an international conference of physicians with expertise in the field to reach a consent. Stage 4 included prospective validation of discovered criteria (63).

The first part of the project was completed in 2010. Questionnaires with 28 clinical, histopathological and laboratory MAS's criteria were sent to physicians from all over the world. The response rate was $45.9 \%$, representing 232 completed questionnaires. Respondents were asked to score the most important diagnostic criteria of MAS; criteria used in practice. The highest average, in order, included the following criteria: Thrombocytopenia, hyperferritinemia, hemophagocytosis, increased liver enzymes, leukopenia, continuous fever, low erythrocyte sedimentation rate (ESR) hypofibrinogenemia and hypertriglyceridemia. Note that 7 of 9 are laboratory criteria and only 1 is a clinical criterion.

The largest median scores obtained the following criteria: Bone marrow hemophagocytosis, hyperferritinemia, fever $\geq 38^{\circ} \mathrm{C}$, thrombocytopenia, low red blood cell sedimentation rate, lactate dehydrogenase elevation, hypofibrinogenemia, and IL-2 soluble receptor growth. In the end, only 9 diagnostic criteria were selected, which received votes from more than $50 \%$ of the surveyed specialists; criteria that will be part of a diagnostic score are documented in Table IV. These are thrombocytopenia, hyperferritinemia, hematopoietic haemophagocytosis, increased liver enzymes, leucopenia, continuous fever, low red blood cell sedimentation rate, hypofibrinogenemia and hypertriglyceridemia. 
Table IV. The 28 items (features) included in the Delphi International Survey, and the percentage of respondents attributing high rank to each feature and mean ranks of features (63).

\begin{tabular}{lcc}
\hline Item & Respondents who selected the feature $\mathrm{n}(\%)$ & Mean (SD) rank \\
\hline Falling platelet count & $201(86.6)$ & $6.1(2.3)$ \\
Hyperferritinemia & $194(83.6)$ & $6.5(3.0)$ \\
Bone marrow hemophagocytosis & $188(81.0)$ & $6.9(3.6)$ \\
Increased liver enzymes & $174(75)$ & $5.0(2.4)$ \\
Falling leukocyte count & $172(74.1)$ & $5.6(2.5)$ \\
Persistent continuous fever $\geq 38^{\circ} \mathrm{C}$ & $158(68.1)$ & $6.0(3.4)$ \\
Falling erythrocyte sedimentation & $142(61.2)$ & $5.5(2.7)$ \\
Hypofibrinogemia & $142(61.2)$ & $5.4(2.4)$ \\
Hypertriglyceridemia & $135(58.2)$ & $5.1(2.7)$ \\
Central nervous system dysfunction & $104(44.8)$ & $5.0(2.9)$ \\
Falling hemoglobin level 100 & $100(43.1)$ & $4.8(2.3)$ \\
Prolongation of clotting times & $81(34.9)$ & $4.5(2.3)$ \\
Increased D-dimer & $76(32.8)$ & $5(2.6)$ \\
Hemorrhagic manifestations & $72(31.0)$ & $5.3(3.0)$ \\
Liver enlargement & $71(30.6)$ & $4.8(2.8)$ \\
Spleen enlargement & $57(24.6)$ & $4.5(2.8)$ \\
Increased lactic dehydrogenase & $45(19.4)$ & $5.5(2.6)$ \\
Increased soluble IL-2 receptor $\alpha$ & $39(16.8)$ & $5.1(3.1)$ \\
Increased soluble CD163 & $27(11.6)$ & $5.2(3.0)$ \\
Lymphadenopathy & $22(9.5)$ & $4.4(2.9)$ \\
Decreased albumin & $19(8.2)$ & $4.3(2.9)$ \\
Hyponatremia & $16(6.9)$ & $5(3.2)$ \\
Arthritis improvement & $14(6.0)$ & $3.1(2.4)$ \\
Renal failure & $13(5.6)$ & $3.6(2.9)$ \\
Jaundice & $9(3.9)$ & $5.9(3.3)$ \\
Increased bilirubin & $9(3.9)$ & $3.4(2.0)$ \\
Respiratory failure & $6(2.6)$ & $4.4(3.0)$ \\
Cardiac failure & $5(2.2)$ & $3.8(2.6)$ \\
\hline
\end{tabular}

The second part of the international collaboration project to create an MAS diagnosis system is still ongoing. Physicians participating in the study collected clinical-paraclinical data from patients who develop MAS as a complication of varous diseases. Data will be collected from the onset and worsening of diseases that can lead to MAS (63).

In January 2011, an interim analysis was conducted, with 76 investigators from 26 countries reporting the following results: 272 MAS patients, 378 rheumatoid but non-MAS patients and 322 patients without rheumatoid disease, but with specific MAS symptoms. Once the data collection is complete, a series of statistical analyses will be carried out. Thus, for each laboratory parameter, the sensitivity and the specificity to be taken into account as a diagnostic criterion of MAS will be calculated. Moreover, combinations of parameters will be performed to test their specificity, sensitivity to create a set of criteria that will differentiate MAS from other diseases with similar manifestations.

The third part of the study refers to the international conference of rheumatologists, pediatricians and hemato-oncologists. The purpose of the summit is to reach a consensus concerning MAS diagnostic criteria using statistical test combinations.
The fourth part of the study will begin after the completion of the MAS diagnostic set criteria, as these will require validation in clinical practice (63).

Finally, Ravelli et al (50) published in 2005 the 'Preliminary Diagnostic Guide for MAS Associated AJIs' in the Journal of Pediatrics. They named 8 criteria that they considered more important in the diagnosis of MAS. Of these, only 5 entered the top 9 criteria chosen by the international consensus of physicians. The criteria included: Thrombocytopenia, increased liver enzymes, leukopenia, hypofibrinogenemia and hemophagocytosis. The other 3 clinical features proposed by Ravelli et al (50) (central nervous system disorders, hemorrhagic diathesis and hepatomegaly) did not have the equivalent in the international study $(47,63)$.

\section{Discussion}

The development of a set of MAS diagnostic criteria is important for the diagnosis of primary macrophage and secondary disease syndrome (infectious, inflammatory, neoplastic). MAS is often subdiagnosed, confused with symptoms of the basic disease, the mortality rate being high $20-53 \%$ (58). 
Table V.Clinical and laboratory features of macrophage activation syndrome according to various authors $(6,18,34,36,50,51,54,61)$.

Percentage of patients affected in the studies:

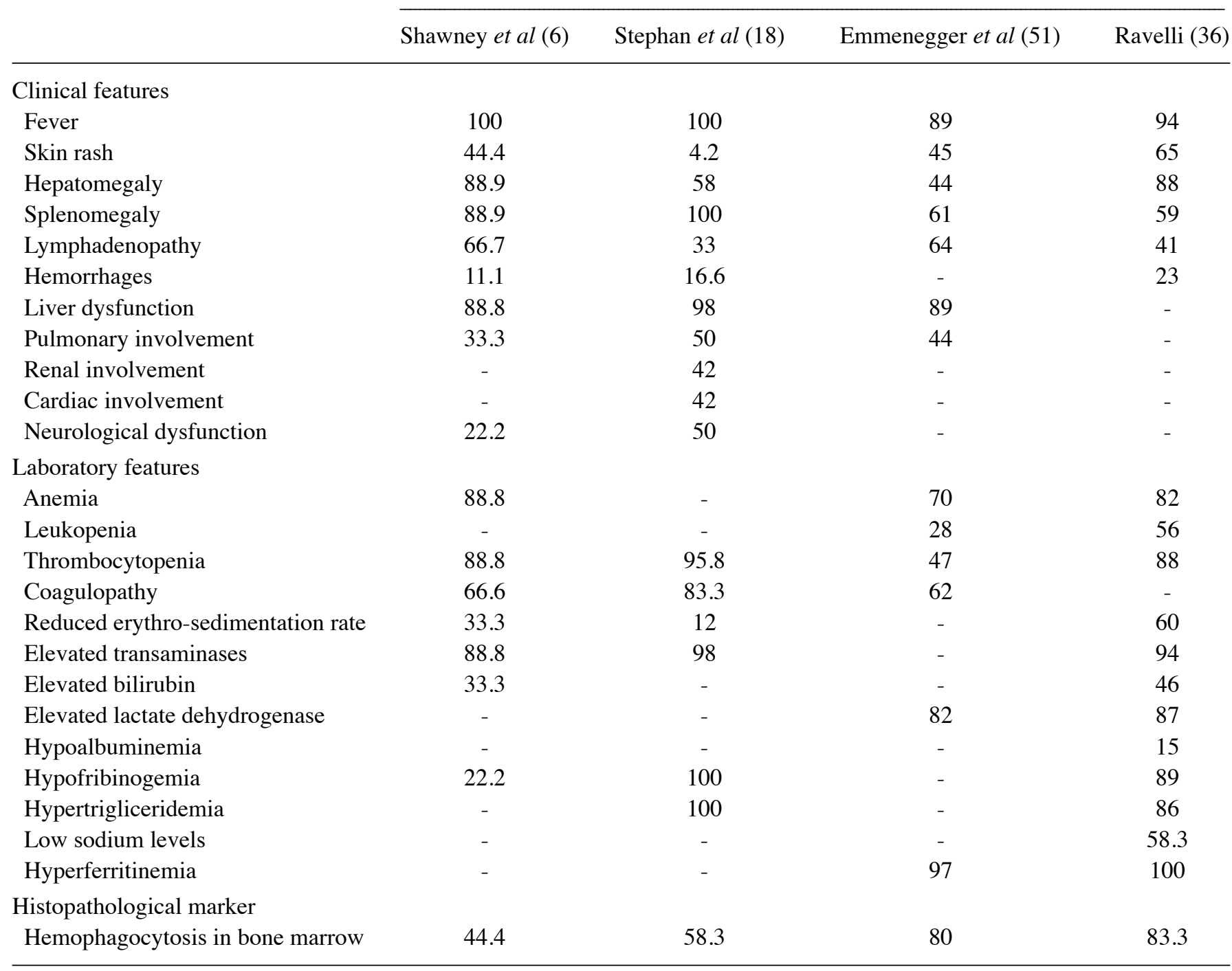

According to the analyzed studies, most MAS diagnostic criteria were paraclinical. The proposed clinical criteria included fever, hepatomegaly, and splenomegaly (5-11,17-21,24-26,29,31,33,35,36,40-47,49-53,57).

Among these criteria, the subsequent parameters that were most commonly used to diagnose MAS were bicitopenia (used in $85 \%$ of studies), fever (used in $55 \%$ of studies), hypofibrinogenemia (used in $55 \%$ of studies), hyperferithemia (used in 55\% of studies), hepatosplenomegaly (used in $55 \%$ of studies), hemophagocytosis (used in $55 \%$ of studies), coagulopathy (used in $55 \%$ of studies), hepatopathy (used in $51.85 \%$ of studies), hypertriglyceridaemia (used in $51.85 \%$ of studies), decrease on erythrocyte sedimentation rate $(22.2 \%)$.

More than half of the analyzed studies cited above used the following diagnostic criteria for MAS: Bicitopenia, fever, hypofibrinogenemia, hyperferritinemia, hepatosplenomegaly, coagulopathy, hemophagocytosis, and hepatopathy.

Decreased erythrocyte sedimentation rate, although a useful criterion for any hospital service, was used by less than half of the authors to diagnose MAS. However, a decrease in erythrocyte sedimentation rate was included in the set of diagnostic criteria proposed by the international Delphi study (63).

Hepatosplenomegaly was present in more than half of the studies analyzed in this literature review without being one of the criteria proposed by the international study.

Most authors proposed criteria that were accessible to nearly all hospitals, with the only less accessible criteria being the increased IL-2 soluble receptor level or the decrease in activity of NK or SCD163 cells.

The most elaborate diagnostic score for MAS was the outcome of the international Delphi study. This study highlighted the importance of diagnostic criteria, according to the opinions of various experts in the field. Sawhney et al (6), Stephan et al (18), Emmenegger et al (51) and Ravelli (36). also outlined sets of diagnostic criteria for MAS, according to each author, some criteria were given more or less importance (Table V) $(6,18,36,50,51,54,58)$.

The limits of this literature review are the limitations in the search for studies which consist of the lack of access to some extended studies provided by the search; the fact that only abstracts were available; lack of access to paid studies; lack of 
inclusion of studies published in languages such as German, French, Spanish, Portuguese, and Japanese.

\section{Conclusions}

MAS is an underdiagnosed acute and severe pathology associated with a high rate of mortality. Being underdiagnosed, MAS is confused with other severe diseases (sepsis, adverse effects of anti-arthritic drugs or exacerbated symptoms of evolving rheumatologic or infectious diseases) because of its biological and clinical polymorphism.

Due to late diagnosis, many patients are not adequately treated leading to a detrimental outcome.

Based on the analysis of the 40 studies included in this review, we conclude that the main diagnostic criteria that should contribute to the development of a diagnostic score for MAS include: Fever, hepatosplenomegaly, hyperferritinemia, hepatopathy, coagulopathy, thrombocytopenia, hypertriglyceridemia, decrease in erythrocyte sedimentation rate and bone marrow hemophagocytosis. The only comprehensive diagnostic score was developed by Davi et al (63) based on an international research project.

These diagnosis criteria could be valuable for many phisicians (immunologists, intensive care specialists, hematologists, infectionists, rheumatologists) in order to recognise this syndrome, and have an effective and accurate treatment for reducing mortality for this cathegory of patients.

\section{Acknowledgements}

Not applicable.

\section{Funding}

No funding was received.

\section{Availability of data and materials}

All information provided in the review is documented by relevant references.

\section{Authors' contributions}

$\mathrm{AB}, \mathrm{AP}$ and ADF contributed substantially to the conception and design of the study, the acquisition, selection, analysis, and interpretation of the data, and were involved in the drafting of the manuscript. IAZ and TT contributed substantially to the acquisition, analysis and interpretation of the data and were involved in the drafting of the manuscript. All authors contributed to the revision of the manuscript. All authors read and approved the final manuscript. AB, AP and ADF made the same contributions in elaborating the article.

\section{Ethics approval and consent to participate}

Not applicable.

\section{Patient consent for publication}

Not applicable.

\section{Competing interests}

The authors declare that they have no competing interests.

\section{References}

1. Lichtman MA, BeutlerE, Thomas JK, Seligsohn U, Kaushansky K and Josef TP: Inflammatory and malignant histiocytosis. In: Williams Hematology. 7th edition. McGraw-Hill Book Co., New York, pp993-1007, 2005.

2. Jordan MB and Filipovich AH: Histiocytic disorders. In: Basic Principles and practice. 6th edition. Elsevier, Philadelphia, PA, pp686-700, 2013

3. Mosser DM and Edwards JP: Exploring the full spectrum of macrophage activation. Nat Rev Immunol 8: 958-969, 2008.

4. Szyper-Kravitz A and Martine M: The hemophagocytic syndrome/macrophage activation syndrome: A final common pathway of a cytokine storm. Isr Med Assoc J 11: 633-634, 2009.

5. Grom AA and Alexei A: Macrophage activation syndrome, a review of diagnosis, treatment, and prognosis. Rheumatologist 4: 22-30, 2012.

6. Sawhney S, Woo P and Murray K: Macrophage activation syndrome: A potentially fatal complication of rheumatic disorders. Arch Dis Child 85: 421-426, 2001.

7. Athreya BH: Is macrophage activation syndrome a new entity? Clin Exp Rheumatol 20: 121-123, 2002.

8. Villanueva J, Lee S, Giannini EH, Graham TB, Passo MH, Filipovich A and Grom AA: Natural killer cell dysfunction is a distinguishing feature of systemic onset juvenile rheumatoid arthritis and macrophage activation syndrome. Arthritis Res Ther 7: 30-37, 2005.

9. Grom AA: Natural killer cell dysfunction: A common pathway in systemic-onset juvenile rheumatoid arthritis, macrophageactivation syndrome, and hemophagocytic lymphohistiocytosis? Arthritis Rheum 50: 689-698, 2004.

10. Canna SW and Behrens EM: Making sense of the cytokine storm: A conceptual framework for understanding, diagnosing, and treating hemophagocytic syndromes. Pediatr Clin North Am 59: 329-344, 2012.

11. Dapena DJL, Díaz de Heredia RC, Bastida VP, Llort SA, Elorza AI, Olivé OT and De Toledo CJS: Haemophagocytic syndrome: A common pathogenic mechanism of various aetiologies. An Pediatr 71: 110-116, 2009.

12. Odegaard JI and Chawla A: Alternative macrophage activation and metabolism. Annu Rev Pathol 6: 275-297, 2011.

13. Strippoli R, Carvello F, Scianaro R, De Pasquale L, Vivarelli M, Petrini S, Bracci-Laudiero L and De Benedetti F: Amplification of the response to Toll-like receptor ligands by prolonged exposure to interleukin-6 in mice: Implication for the pathogenesis of macrophage activation syndrome. Arthritis Rheum 64: 1680-1688, 2012.

14. Behrens EM, Canna SW, Slade K, Rao S, Kreiger PA, Paessler M, Kambayashi T and Koretzky GA: Repeated TLR9 stimulation results in macrophage activation syndrome-like disease in mice. J Clin Invest 121: 2264-2277, 2011.

15. Grom AA, Villanueva J, Lee S, Goldmuntz EA, Passo MH and Filipovich A: Natural killer cell dysfunction in patients with systemic-onset juvenile rheumatoid arthritis and macrophage activation syndrome. J Pediatr 142: 292-296, 2003.

16. Clementi R, Emi L, Maccario R, Liotta F, Moretta L, Danesino C and Arico M: Adult onset and atypical presentation of hemophagocytic lymphohistiocytosis in siblings carrying PRF1 mutations. Blood 100: 2266-2267, 2002.

17. Bracaglia C, Prencipe G and De Benedetti F: Macrophage activation syndrome: Different mechanisms leading to a one clinical syndrome. Pediatr Rheumatol Online J 15: 5, 2017.

18. Stephan JL, Zeller J and Hubert P: Macrophage activation syndrome and rheumatic disease in childhood: A report of four new cases. Clin Exp Rheumatol 11: 451-456, 1993.

19. You CR, Kim HR, Yoon CH, Lee SH, Park SH and Kim HY: Macrophage activation syndrome in juvenile rheumatoid arthritis successfully treated with cyclosporine A: A case report. J Korean Med Sci 21: 1124-1127, 2006.

20. Kelly A and Ramanan AV: Recognition and management of macrophage activation syndrome in juvenile arthrititis. Curr Opin Rheumatol 9: 477-481, 2007.

21. Deane S, Selmi C, Teuber SS and Gershwin ME: Macrophage activation syndrome in autoimmune disease. Int Arch Allergy Immunol 153: 109-120, 2010. 
22. Bennett TD, Fluchel M, Hersh AO, Hayward KN, Hersh AL, Brogan TV, Srivastava R, Stone BL, Korgensky EK, Mundorff MB, et al: Macrophage activation syndrome in children with systemic lupus erythematosus and children with juvenile idiopathic arthritis. Arthritis Rheum 64: 4135-4142, 2012.

23. Shafferman A, Birmingham JD and Cron RQ: High dose anakinra for treatment ofsevere neonatal Kawasaki disease: A case report. Pediatr Rheumatol Online J 11: 12-26, 2014.

24. Egües DCA, Uriarte EM, Meneses VC, Aldasoro CV, Hernando RI and Belzunegui OJ: Hemophagocytic syndrome as the initialmanifestation of systemic lupus erythematosus. Reumatol Clin 10: 321-324, 2014.

25. Vishwanath VK, Krishnamurthy A, Karyampudi A and Dutta TK: Fever of unknown origin in a patient of systemic onset juvenile idiopathic arthritis. Indian J Med Sci 64: 333-336, 2010

26. Young LR, Borchers MT, Allen HL, Gibbons RS and McCormack FX: Lung-restricted macrophage activation in the pearl mouse model of Hermansky-Pudlak syndrome. J Immunol 176: 4361-4368, 2006.

27. Hur M, Kim YC, Lee KM and Kim KN: Macrophage activation syndrome in a child with systemic juvenile rheumatoid arthritis. J Korean Med Sci 20: 695-698, 2005.

28. Boca AN, Talamonti M, Galluzzo M, Botti E, Vesa SC, Chimenti S, Buzoianu AD and Costanzo A: Genetic variations in ILB decreasing the risk for psoriasis. Immunol Lett 156: 127-131, 2013.

29. Liu AC, Yang Y, Li MT, Jia Y, Chen S, Ye S, Zeng XZ, Wang Z, Zhao JX, Liu XY, et al: Macrophage activation syndrome in systemic lupus erythematosus: A multicenter, case-control study in China. Clin Rheumatol 37: 93-100, 2018.

30. Cascio A, Pernice LM, Barberi G, Delfino D, Biondo C, Beninati C, Mancuso G, Morales AJR and Iaria C: Secondary hemophagocytic lymphohistiocytosis in zoonoses. A systematic review. Eur Rev Med Pharmacol Sci 16: 1324-1337, 2012.

31. Ruiz GMA, Ruiz JA, Menéndez GJL,Pérez CAM,Domínguez MS and Rodríguez EF: Visceral leishmaniasis and bronchial asthma: Influence of steroid therapy in the development of the macrophage activation syndrome and relative adrenal insufficiency. An Med Interna 25: 279-283, 2008.

32. Soldo-Juresa D, Radman M, Pejsa V and Bozikov V: Reactive macrophage activation syndrome in a patient with parvovirus B19 infection, lymphocytic lichenoid vasculitis, urticaria and angioedema. Ann Saudi Med 30: 88-89, 2010.

33. Chellapandian D, Das R, Zelley K, Wiener SJ, Zhao H, Teachey DT and Nichols KE; EBV-HLH Rituximab Study Group: Treatment of Epstein Barr virus-induced haemophagocytic lymphohistiocytosis with rituximab-containing chemo immunotherapeutic regimens. Br J Haematol 162: 376-382, 2013.

34. Stabile A, Bertoni B, Ansuini V, La Torraca I, Salli A and Rigante D: The clinical spectrum and treatment options of macrophage activation syndrome in the pediatric age. Eur Rev Med Pharmacol Sci 10: 53-59, 2006.

35. Kuppe C, Westphal S, Bücher E, Moeller MJ, Heintz B, Schneider ME and Floege J: Macrophage activation syndrome in a patient with pulmonary inflammatory myofibroblastic tumour. Allergy Asthma Clin Immunol 8: 6, 2012.

36. Ravelli A: Macrophage activation syndrome. Curr Opp Rheum 14: 548-552, 2002.

37. Souabni L, Dridi L, Ben AK, Kassab S, Chekili S, Laater A and Zakraoui L: Possible macrophage activation syndrome following initiation of adalimumab in a patient with adult-onset Still's disease. Pan Afr Med J 17: 94, 2014

38. Ikegawa T, Yamazaki K, Nishimura K, Kanetaka T, Kikuchi M, Nozawa T, Hara R, Sato T, Sakurai N and Yokota S: A case of severe systemic juvenile idiopathic arthritis introduced tocilizumab in early phase of the disease. Nihon RinshoMeneki Gakkai Kaishi 37: 176-182, 2014.

39. Delavigne K, Bérard E, Bertoli S, Corre J, Duchayne E, Demur C, De Mas VM,Borel C,Picard M, Alvarez M, et al: Hemophagocytic syndrome in patients with acute myeloid leukemia undergoing intensive chemotherapy. Haematologica 99: 474-480, 2014.

40. Sandhu C, Chesney A, Piliotis E, Buckstein R and Koren S Macrophage activation syndrome after etanercept treatment. J Rheumatol 34: 241-242, 2007.

41. Teachey DT, Rheingold SR, Maude SL, Zugmaier G, Barrett DM Seif AE, Nichols KE, Suppa EK, Kalos M, Berg RA, et al: Cytokine release syndrome after blinatumomab treatment related to abnormal macrophage activation and ameliorated with cytokine-directed therapy. Blood 121: 5154-5157, 2013.
42. Leslye FC, Aedo PK and Miraval-Niño de Guzmán T: Macrophage activation syndrome: Experience in the questioned role of etoposide. Reumatol Clin 13: 239-240, 2017 (In English, Spanish).

43. Hadchouel M, Prieur AM and Griscelli C: Acute hemorrhagic, hepatic, and neurologic manifestations in juvenile rheumatoid arthritis: Possible relationship to drugs or infection. J Pediatr 106: 561-566, 1985

44. Janka G and Zur Stadt U: Familial and acquired hemophagocytic lymphohistiocytosis. Hematology Am Soc Hematol Educ Program: 82-88, 2005 doi: 10.1182/asheducation-2005.1.82.

45. Ramanan AV and Baildam EM: Macrophage activation syndrome is hemophagocytic lymphohistiocytosis-need for the right terminology. J Rheumatol 29: 1105, 2002.

46. Haque SF: Macrophage activation syndrome. Med Update 7: 447, 2011.

47. Davì $S$, Lattanzi $B$, Demirkaya $E$, Rosina $S$, Bracciolini $S$, Novelli A, Ruperto N, Martini A, Cron R and Ravelli A: Toward the development of new diagnostic criteria for Macrophage activation syndrome in Systemic juvenile idiopathic arthritis. Ann Paediatr Rheum 1: 1-7, 2012.

48. Grom AA: Macrophage activation syndrome and reactive hemophagocytic lymphohistiocytosis: The same entities? Curr Opin Rheumatol 15: 587-590, 2003.

49. Ramanan AV and Schneider R: Macrophage activation syndrome-what's in a name!. J Rheumatol 30: 251-253, 2003.

50. Ravelli A, Magni-Manzoni S, Pistorio A, Besana C, Foti T, Ruperto N, Viola S and Martini A: Preliminary diagnostic guidelines for macrophage activation syndrome complicating systemic juvenile diopathic arthritis. Pediatr 146: 598-604, 2005.

51. Emmenegger U, Schaer DJ, Larroche C and Neftel KA Haemophagocytic syndromes in adults: Current concepts and challenges ahead. Swiss Med Wkly 135: 299-314, 2005.

52. Feldmann J, Callebaut I and Raposo G: Munc 13-4 is essential for cytolytic granules fusion and is mutated on a form of familial hemophagocytic lymphohistiocytosis (FHL3). Cell 115: 461-473, 2003.

53. Rosado FG and Kim AS: Hemophagocytic lymphohistiocytosis: An update on diagnosis and pathogenesis. Am J Clin Pathol 139: 713-727, 2013.

54. Stephan J, Kone-Paut I, Galambrun C, Mouy R, Bader-Meunier B and Prieur A: Reactive haemophagocytic syndrome in children with inflammatory disorders: A retrospective study of 24 patients. Rheumatology 40: 1285-1292, 2001.

55. Canna SW and Behrens EM: Not all hemophagocytes are created equally: Appreciating the heterogeneity of the hemophagocytic syndromes. Curr Opin Rheumatol 24: 113-118, 2012.

56. Bode FN, Ammann S, Al-Herz W, Bataneant M, Dvorak CC, Gehring S, Gennery A, Gilmour KC, Gonzalez-Granado LI, Groß-Wieltsch U, et al: The syndrome of hemophagocyticlymphohistiocytosis in primary immunodeficiencies: Implications for differential diagnosis and pathogenesis. Haematologica 100: 978-988, 2015

57. Lenghel LM, Baciut G, Botar-Jid C, Vasilescu D, Bojan A and Dudea SM: Ultrasonographic identification of the anatomical landmarks that define cervical lymph nodes spaces. Med Ultrason 15: 29-34, 2013.

58. Emmenegger U, Reimers A and Frey U: Reactive macrophage activation syndrome: A simple screening strategy and its potential in early treatment initiation. Swiss Med Wkly 132: 230-236, 2002.

59. Ramanan AV, Akikusa JD, Silverman ED and Schneider R: Extreme hyperferitinemia in childhood. Clin Exp Rheumatol 21: 55-59, 2003.

60. Gorelik M, Fall N, Altaye M, Barnes MG, Thompson SD, Grom AA and Hirsch R: Follistatin-like protein 1 and the ferritin/erythrocyte sedimentation rate ratio are potential biomarkers for dysregulated gene expression and macrophage activation syndrome in systemic juvenile idiopathic arthritis. J Rheumatol 40: 1191-1199, 2013.

61. Emmenegger U, Frey U and Reimers A: Hyperferritinemia as indicator for intravenous immunoglobulin treatment in reactive macrophage activation syndromes. Am J Hematol 68: 4-10, 2001.

62. Orasan OH, Iancu M, Sava M, Saplontai-Pop A, Cozma A, Sarlea ST, Lungoci C, Ungureanu MI, Negrean V, Sampelean D and Dumitrascu DL: Non-invasive assessment of liver fibrosis in chronic viral hepatitis. Eur J Clin Invest 45: 1243-1251, 2015.

63. Davi S, Consolaro A, Guseinova D, Pistorio A, Ruperto N, Martini A, Cron RQ and Ravelli A; MAS Study Group: An international consensus survey of diagnostic criteria for macrophage activation syndrome in systemic juvenile idiopathic arthritis. J Rheumatol 38: 764-768, 2011.

This work is licensed under a Creative Commons Attribution-NonCommercial-NoDerivatives 4.0 International (CC BY-NC-ND 4.0) License. 